\title{
La odisea de un concepto nómada: el desarrollo sustentable en la órbita de la geografía
}

\author{
Dr. Vincent Clément ${ }^{1}$
}

\begin{abstract}
RESUMEN
Los principios del desarrollo sustentable se articulan alrededor de un tríptico formado por la durabilidad medio ambiental, la viabilidad económica y la equidad social. Sin embargo, en cada ámbito relacionado con el tema, el contenido y los objetivos dados a este concepto pueden ser sensiblemente diferentes. ¿Para la investigación en geografía, que postura es conveniente adoptar? ¿El desarrollo sustentable, como concepto nómada, puede convertirse en una herramienta innovadora? ¿O su incertidumbre semántica nos conduce de manera inexorable hacia un vagabundeo de las ideas? El objetivo central de este artículo es intentar situar el concepto de desarrollo sustentable en la órbita de la geografía, en una óptica comparativa con disciplinas vecinas. Para ello, es necesario acercarse sin tabúes a los problemas planteados por este concepto y analizar en que medida la geografía podría enriquecer las reflexiones sobre este nuevo paradigma.

Palabras claves : desarrollo sustentable, concepto nómada, geografía de las utopías, ciudad sustentable, bosque sustentable

ABSTRACT

Abstract. The concept of sustainable development is has developed around the interrelated principles of environmental durability, economic viability and social equity. Nevertheless, each of these three analytical fields is subject to often divergent objectives, assumptions and outcomes. In the discipline of geography, for instance, which category ought to take priority? Can sustainable development, as a nomadic concept, be transformed into a tool for real innovation, or will its semantic uncertainty render the concept of little value, a virtual hodge-podge of disparate ideas and values. The central objective of this article is to situate the concept of sustainable development within the geographical orbit, but in a comparative and interdisciplinary optic. In order to achieve this goal, it is necessary to approach sustainable development and the problems it presents without taboos, and analyze the potential for geography to enrich our shared reflections on this emerging paradigm.
\end{abstract}

Key Words : sustainable development, nomadic concept, utopian geography, sustainable cities, sustainable forests.

Recepcionado: abril 2005

Aceptado: julio 2005

${ }^{1}$ Doctor en Geografía y antiguo miembro científico de la Casa de Velázquez (Madrid), Profesor titular de Geografía École Normale Supérieure LSH, Departamento de Geografía, 15 Parvis René Descartes 69342 Lyon Cedex 07, Francia, vclement@ens-Ish.fr 


\section{INTRODUCCIÓN}

Nuevo paradigma para algunos, o simple eslogan político-mediático para otros, el concepto de desarrollo sustentable levanta reacciones contradictorias en el mundo científico. Tal situación surge de la naturaleza misma de este concepto que navega entre diferentes campos de pensamiento. Bien es sabido que los principios del desarrollo sustentable se articulan alrededor de un tríptico formado por la durabilidad medio ambiental, la viabilidad económica y la equidad social (Brundtland Report, 1987). Sin embargo, en cada ámbito relacionado con el tema, los contenidos y los objetivos dados a este concepto pueden ser sensiblemente diferentes. Los actores políticos ven en ello sobre todo su dimensión ética y la oportunidad de promover una nueva conciencia ciudadana. Los economistas lo entienden como un modelo económico alternativo, menos dilapidador de los recursos naturales. Para el movimiento ecologista, constituye el fundamento de la conservación de la naturaleza. Para los sociólogos, el desarrollo sustentable conlleva un conjunto de valores positivos, una concepción renovada de la idea de progreso que podría ser el inicio de un renacimiento humanista (Jollivet, 2001, p. 9 ; Kalaora, 2005, p. 158).

Este carácter nómada del concepto (Stengers, 1987) de desarrollo sustentable, al menos en Francia, es percibido por ciertos investigadores en geografía como una verdadera tara genética, cuando otros piensan por el contrario que puede servir de palanca para renovar el acercamiento geográfico a los grandes problemas contemporáneos. ¿Para la investigación en geografía, qué postura es conveniente adoptar? ¿El desarrollo sustentable, como concepto nómada, puede convertirse en una herramienta eficaz e innovadora? ¿O bien su incertitumbre semántica nos conduce de manera inexorable hacia un vagabundeo de las ideas?

El objetivo central de esta contribución es intentar situar el concepto de desarrollo sustentable en la órbita del pensamiento geográfico, en una óptica comparativa con disciplinas vecinas. Para ello, es necesario acercarse sin tabúes a los problemas planteados por este concepto y analizar en que medida la geografía podría enriquecer las reflexiones sobre este nuevo paradigma.

\section{El pecado del nomadismo original}

¿Debe considerarse el nomadismo conceptual del desarrollo sustentable como una especie de pecado original que lo convertiría en un instrumento inoperante para la investigación en geografía? Para responder a esta pregunta, es necesario recordar que el concepto de desarrollo sustentable aparecio primero en el mundo político. Este hecho no es para nada anódino. Determinó una cierta desconfianza, al menos en un principio, por parte de la comunidad científica. Luego, surgieron dudas más concretas a la hora de utilizarlo como objeto de ciencia, en particular por la opacidad que encubre sus dimensiones espaciales y temporales.

\section{El nacimiento del concepto}

Brevemente, porque es un aspecto bastante conocido, conviene recordar que el informe Brundtland fue el acta de nacimieno del concepto de desarrollo sustentable. Con el título "Our Common Future", fue presentado en 1987 en las Naciones Unidas por Gro Harlem Brundtland, entonces primera ministra de Noruega y presidenta de la Comisión Mundial para el Medio Ambiente y el Desarrollo de la ONU. Fue luego mediatizado y popularizado en la Cumbre de la Tierra de 1992 celebrado en Río de Janeiro dónde fue adoptado un plan de acción conocido como la "Agenda 21 " (Ducroux, 2002, p. 14). Los autores del informe preconizaban un desarrollo que permitiera satisfacer las necesidades del presente, pero sin comprometer la capacidad de las futuras generaciones en satisfacer las suyas.

Partiendo de una evaluación poco halagüeña del estado del planeta (desertificación, deforestación, disminución de la biodiversidad, contaminación, etc.), el objetivo de los autores era 
establecer un nuevo contrato social entre el hombre y la naturaleza sintetizado en la expresión de " sustainable development ". El éxito de esta hábil construcción semántica es incontestable. El desarrollo sustentable se ha impuesto a lo largo de los años 1990 en todos los debates sobre las cuestiones medioambientales, tanto al nivel mundial como local.

En efecto, en muchos países a través del mundo, se adoptaron medidas legislativas para fundar jurídicamente las políticas nacionales y regionales a favor del desarrollo sustentable. En Chile por ejemplo, la ley de 1994 sobre el Medio Ambiente define el desarrollo sustentable como : " el proceso de mejoramiento sostenido y equitativo de la calidad de vida de las personas, fundado en medidas apropiadas de conservación y protección del medioambiente, de manera de no comprometer las expectativas de las generaciones futuras " (Ley $n^{\circ} 19.300$, letra G, artículo $2^{\circ}$ ). Esto implica promover políticas no solamente a corto plazo, sino más bien con una visión a largo plazo (Hoffmann, 2001, p. 282).

¿Frente al éxito del desarrollo sustentable, cómo explicar las reticencias o las críticas de una franja importante de la comunidad científica en contra a un concepto que ha sido bien acogido en el mundo político y en los grandes medios de comunicación?

\section{Las dudas de los científicos}

Una primera explicación podría ser la desconfianza natural de los investigadores hacia lo que fue percibido en un principio como un fenómeno de moda. El hecho de que este concepto viniera del mundo político, y que fuera tan mediatizado, ha contribuido a alimentar una actitud de prudente reserva por parte de la comunidad científica, en particular por los representantes de las ciencias humanas.

Otra explicación está relacionada con el nomadismo del concepto de desarrollo sustentable.
Nacido en el ámbito de los grandes organismos internacionales, fue concebido ante todo como un proyecto político, y no como un objeto de ciencia.

De ahí su caracter generalizador, plástico, sin contornos bien establecidos. Esto constituye sin duda su fuerza, ya que su difusión en el mundo se ha hecho realidad, más allá de las diferencias culturales o linguísticas. Pero es también lo que podríamos Ilamar su pecado original, puesto que al no tener una definición clara, con límites palpables, puede recubrir significados variables, convirtiéndose en un concepto maleable y por lo tanto manipulable. Demasiado consensual, se aparenta a lo que los lingüistas Ilaman un concepto maleta, capaz de transportar un amplio abanico de ideas y de representaciones a veces contradictorias.

El nomadismo conceptual del desarrollo sustentable, y su aspecto globalizador, hacen que casi todas las disciplinas académicas estén convocadas al mismo tiempo (filosofía, ética, política, economía, ecología, sociología, historia, geografía, ingienería, etc.), situación al menos insólita. Por lo general, los conceptos utilizados por los científicos suelen nacer en un determinado campo disciplinario para luego, después de un cierto tiempo de maduración y de convalidación, extenderse hacia disciplinas vecinas. La simultaneidad de la reflexión sobre el desarrollo sustentable en todos los campos del pensamiento, impuesta en gran parte por el carácter instantáneo de la difusión de la información en nuestra sociedad de la comunicación, representa pues una dificultad suplementaria. Es fácil de comprender que un ecólogo, un sociólogo o un ingeniero, no tienen la misma manera de abordar la problemática del desarrollo sustentable (Martin, 2002). El planteamiento del desarrollo sustentable es ciertamente global. Pero las aportaciones de la comunidad científica responden a un sistema de pensamiento, a herramientas conceptuales y a prácticas de investigación propias de cada disciplina, sobre todo cuando se trata de dar contenido a un concepto nuevo y de situarlo en una filiación epistemológica. 


\section{El espacio y el tiempo}

En el caso de la geografía, el desarrollo sustentable padece de una referencia insuficiente a dos dimensiones muy arraigadas a nuestra disciplina, el espacio y el tiempo. La casi ausencia de referencias espaciales en este concepto es un serio obstáculo para la investigación. El leitmotiv de la Agenda 21, "Pensar globalmente, actuar localmente", es la única referencia espacial, por lo menos esquemática, entre la escala planetaria y la escala local. Como geógrafo, este eslogan sólo puede darnos vértigo, ya que borra sin más todo el juego complejo de las escalas, omitiendo en particular las escalas intermedias. Tal lema puede ser incluso peligroso. ¿Quién tendría el papel de pensar globalmente? ¿Los expertos de los grandes organismos internacionales? ¿Qué rol tendrían los actores locales? ¿Se convertirían en simples ejecutantes de un pensamiento único, impuesto sin matices a todo el planeta?

Aunque el desarrollo sustentable se inscriba en una toma de conciencia planetaria, es sin duda a escala regional y local donde serán elaboradas las políticas más eficaces. Por lo tanto, es probablemente oportuno darle la vuelta al eslogan de la Agenda 21, defendiendo la idea de que sería preferible pensar localmente, para actuar globalmente. En otras palabras, sería más pertinente inventar políticas y prácticas de desarrollo sustentable a nivel regional y local, eso sí, persiguiendo un objetivo común, el de un desarrollo más respetuoso del medio y de los recursos naturales.

De la misma forma, el tema de la temporalidad, en el corazón mismo de la noción de durabilidad, está paradójicamente eludido de la reflexión sobre el desarrollo sustentable. Este concepto implica una responsabilidad hacia las futuras generaciones, pero sin que sepamos realmente sobre qué escala temporal nos situamos. ¿De qué generaciones se trata : las de 2025, de 2050 , de 2100 o de más allá? El compromiso con las generaciones futuras levanta otros interrogantes. ¿Podemos realmente anticipar las necesidades $y$ las esperanzas de la generaciones futuras? ¿No sería eso una forma de negar, de manera consciente o no, su capacidad de innovación? Otra dificultad conlleva el que el concepto de desarrollo sustentable nos impone un eje del tiempo único y lineal, orientado hacia el futuro y negando de hecho las herencias del pasado. ¿Cómo puede ser contemplada la idea de proyectarse hacia el futuro, sin tener en cuenta las relaciones pasadas entre las sociedades y su entorno natural? ¿En esta visión única y lineal del tiempo, cómo articular los fenómenos que se desarrollan sobre diferentes escalas temporales (tiempos de las sociedades, del medio ambiente, de la economía, de la política.), así como los que corresponden a lógicas temporales no lineales (fenómenos cíclicos, crisis y perturbaciones, variabilidad climática)?

Sin duda, el desarrollo sustentable ilustra la dificultad en traspasar la frontera entre lo político y lo científico. Los investigadores necesitan tiempo para adoptar un concepto y poder cuestionarlo para convertirlo en una herramienta conceptual. Al contrario, los actores políticos piden respuestas inmediatas. Esto es poco compatible con las exigencias de la investigación científica y menos aún cuando surgen incógnitas sobre el origen de un nuevo concepto.

\section{Bajo la máscara de la novedad}

El desarrollo sustentable ha sido presentado con la vestimenta atractiva de la novedad, como un concepto sacado de ninguna parte, un puro invento surgido de la mente luminosa de los expertos de la ONU. ¿Sería el desarrollo sustentable tan innovador, tan original, tan inesperado que, al contrario de la inmensa mayoría de los conceptos, sería también huérfano de toda filiación intelectual, apartado de la historia de las ideas, marcando una ruptura radical con todo lo conocido hasta ahora? 


\section{Una idea antigua}

A esta pregunta, hay que responder con un no rotundo. Lo de preservar los recursos naturales, de llevar a cabo una gestión concienzuda de la naturaleza, de limitar los efectos negativos de la sobre-explotación humana y de proyectarse hacia el futuro, es en realidad una idea antigua. Que las medidas tomadas antiguamente para conseguirlo hayan sido exitosas o no, es otra cuestión.

Es en la legislación medieval europea sobre los bosques donde se encuentran las primeras normativas para conservar este recurso entonces estratégico, pues era un material indispensable para la edificación, la construcción de barcos, la calefacción y demás. En Francia y en España, el poder real en el siglo XIV vio de forma muy clara la necesidad de preservar los bosques. En el país galo, la Ordenanza de Brunoy, promulgada por Felipe VI de Valois en 1346, ilustra esta voluntad política. en su artículo 4 , se estipula que los maestres de bosques inspeccionaran regularmente todas las forestas del reino, para que "los dichos bosques puedan mantenerse perpetuamente en buen estado" (Arnould y Clément, 2004, p. 168). Pocos años después, Pedro I de Trastamara, en las Cortes de Valladolid de 1351 , denunció con vehemencia las talas abusivas de los bosques en Castilla en estos términos: "[...] en las çibdades e villas e lugares delos mios rreynos se destruyen de cada día de mala manera los montes, senalada miente los pinares e enzinares, porque derriban çinco o sseys pinnos para tirar dende tres o quatro rrayos de tea que non valen tres dineros, et que en los enzinares, por un palo muy ssotil que ayan meester, que cortan un enzina por pie [...]". (Real Academia de la historia, p. 36).

Esta cita nos revela ciertamente los abusos cometidos en esta época, pero también la relevancia del tema de la preservación de los bosques para Pedro I. Las medidas tomadas por el rey estuvieron a la altura de su enfado y de la imperiosa necesidad de salvaguardar los recursos forestales: "[...] Et qual quier que cortare o derraygare o quemare pinnos en los pinares o enzinas en los encinares quel maten por ello e demas que pierda todos sus bienes [...]" (Real Academia de la historia, p. 36).

La búsqueda de un equilibrio entre la explotación de los bosques y su preservación para el futuro no fue solamente una preocupación de soberanos, como lo atestiguan las innumerables ordenanzas forestales locales del medievo en Castilla (Clément, 2002, p. 243). Así por ejemplo, las ordenanzas forestales de 1492 de la Comunidad de Villa y Tierra de Cuéllar, territorio que contaba con importantes masas de pinares, impusieron una serie de disposiciones para gestionar de manera duradera este recurso natural, fijándose como meta la conservación y el aumento de los bosques, lo que consiguieron hasta hoy día. Por lo tanto, fue en el medievo cuando apareció el primer zócalo jurídico de lo que en la actualidad Ilamamos el desarrollo sustentable (Arnould y Clément, 2004, p. 168-169).

En el Siglo de las Luces y en el XIX, numerosos pensadores (filósofos, economistas, geógrafos, etc.) formularon con más fundamentos teóricos la necesidad de una mejor adecuación entre la explotación de la naturaleza y su conservación. En 1798, Malthus, en su Ensayo sobre el principio de la población, preconizaba limitar o controlar el crecimiento de la población para no comprometer la sustentabilidad de los recursos naturales (Robinson, 2002, p. 42). Medio siglo más tarde, en 1848, el economista inglès John Stuart Mill defendió por primera vez la idea de un estado estacionario de la economía (lo que hoy se conoce como la teoría del crecimiento cero) para preservar la naturaleza, en una óptica humanista y utilitaria. O'Connor ha interpretado la idea de J. Stuart Mill como la base de una nueva ética medioambiental, aunque tal idea no fuera tan claramente formulada por el economista ingles (O'Connor, 1995). A su vez, el geógrafo americano George Perkins Marsh (1864), subrayó el carácter absurdo del desperdicio de los recursos naturales, hecho que consideraba contrario a la voluntad de Dios y a los intereses vitales de las naciones. El filósofo francés, Antoine Augustin Cournot, preveía en 1861, con un cierto 
grado de catastrofismo, que la destrucción por el hombre de la naturaleza anunciaba el final del progreso de la humanidad (Vivien, 2001, p. 40-41).

\section{Del ecodesarrollo al desarrollo sustentable}

Más recientemente, a finales de los años 1960 y al inicio de la década de los 1970 , con la creación del Club de Roma, la Declaración de Estocolmo sobre el Hombre y el Medio Ambiente en 1972 y la publicación del Informe Meadows el mismo año, el acercamiento al concepto de desarrollo sustentable se hizo aún más preciso. Los autores del informe Meadows rehabilitaron la idea de un crecimiento cero (el estado estacionario de John Stuart Mill), para remediar el mal desarrollo y la escasez de los recursos naturales (Meadows, 1972). Frente al modelo de desarrollo dominante, dilapidador de los recursos naturales y provocando a veces daños irremediables sobre el medio ambiente, el economista Ignacy Sachs propuso un contra-modelo, el del "ecodesarrollo", orientado hacia un desarrollo endógeno y capaz de preservar los recursos naturales (Sachs, 1977, p. 9-10).

La crisis del petróleo de 1973 dio crédito a esta nueva visión de la relación entre economía y medio ambiente (Pisani, 2005, p. 30). Pero el concepto de ecodesarrollo tuvo poca incidencia en la práctica, porque apareció demasiado opuesto al modelo económico dominante en aquel momento. Ese no fue el destino del concepto de desarrollo sustentable, por al menos tres razones. En primer lugar, porque su formulación es bastante más consensual. En segundo lugar, porque se beneficia de un interés mayor sobre las cuestiones medioambientales por parte de los actores políticos y de los ciudadanos en los años 1970. Y en tercer lugar, porque el concepto de desarrollo sustentable tiene una mayor visibilidad, gracias al papel desempeñado por las ONGs ambientalistas, el movimiento anti-globalización y los grandes medios de comunicación.

Así que el concepto de desarrollo sustentable, lejos de ser una idea muy nueva, se inscribe en una filiación intelectual antigua, y más directamente en las teorías del siglo XIX y los análisis de los años 1970 que tenían como finalidad una mejor adecuación entre economía, desarrollo y medio natural. Sin embargo, el desarrollo sustentable no es una mera nueva forma de expresar las ideas de los años 1970, que eran en gran parte fundadas sobre criterios cuantitativos. Se sitúa en un acercamiento más cualitativo de la problemática, introduciendo una dimensión ética que aparecía sólo perfilada en el concepto de ecodesarrollo. Introduce también una proyección hacia el futuro, lo que representa ciertamente un desafío para la ciencia. ¿Cómo integrar, en la investigación geogáfica, la problemática del desarrollo sustentable y el principio de incertidumbre inherente a este concepto?

\section{Hacia una geografía de las utopías}

Más allá de los interrogantes que levanta el concepto de desarrollo sustentable, no se puede negar que la idea de durabilidad es uno de los paradigmas del pensamiento geográfico (Robic $y$ Mathieu 2001, p. 167-168). Desde el siglo XIX y a lo largo del siglo XX, los temas clásicos de nuestra disciplina se centraron en gran parte sobre las relaciones más duraderas entre el hombre y su entorno (habitabilidad de la tierra, cuadros y géneros de vida, paisajes, humanización del medio natural). Sin embargo, es necesario una cierta introspección disciplinaria para ver en que medida la geografía puede alimentar la reflexión sobre el desarrollo sustentable.

\section{Sustentabilidad y geografía}

En el coloquio de la Unión Geográfica Internacional sobre el desarrollo sustentable, celebrado en Glasgow en agosto 2004, las comunicaciones cubrieron un amplio panel de temas, como se puede ver por ejemplo en la síntesis 
presentada por el Comité Nacional Francés de Geografía (Miossec, Arnould, Veyret, 2004). Resalta de estas comunicaciones que es en el campo de la geografía medioambiental donde se encuentra la filiación más evidente entre nuestra disciplina y el desarrollo sustentable.

En una perspectiva epistemológica, podemos decir que la evolución de nuestra disciplina sobre el hombre y su entorno natural estuvo basada en conceptos diferentes y que cada nuevo eslabón conceptual marcó un paulatino deslizamiento desde el polo de la naturaleza hacia el polo de la cultura. El concepto clásico de "medio" fue el pilar conceptual de la geografía naturalista en la cual el hombre era marginalizado, o bien aparecía como un elemento perturbador de sistemas definidos ante todo sobre leyes naturales. A partir de los años 1970, los conceptos de "medio ambiente" y de "geosistema" expresaron un giro importante: al resituar el análisis del medio en su contexto social e histórico, orientaron la investigación hacia un mejor equilibrio entre naturaleza y cultura (Bertrand, 2002). Con el desarrollo sustentable, la geografía ambientalista podría dar un paso más. La dimensión indudablemente ética del concepto (Thompson, 2000, p. 13-14), con su compromiso con las futuras generaciones, debería colocar al hombre en el punto de partida de la investigación medioambiental en geografía.

Ahora bien, para hacer del desarrollo sustentable un verdadero objeto de ciencia, hay que tener presente que este concepto superpone diferentes utopías: una utopía ética (el renacimiento humanista), una utopía económica (un modelo de desarrollo alternativo), una utopía ecologista (la preservación del medio natural), una utopía geopolítica (establecer relaciones Norte/Sur más equilibradas). El punto común de estas utopías es otra utopía, la de salvaguardar el planeta para las generaciones futuras. Así que investigar sobre el desarrollo sustentable nos conduce a abrir un nuevo frente en nuestra disciplina, el de una geografía de lo incierto, de lo posible o, en suma, lo que podríamos Ilamar una geografía de las utopías. ¿Qué sentido, como geógrafos, podemos dar a esta superposición de utopías? ¿El de una meta a alcanzar, o bien el de una vía que orienta el pensamiento y la acción sobre los territorios? 2. La utopía de la ciudad sustentable

El ejemplo del debate actual sobre la ciudad sustentable permite abordar de forma más concreta tales interrogantes. La masiva adhesión de las ciudades europeas a la Carta de Alborg sobre la ciudad sustentable (1994), adoptada por cerca de 1500 ciudades en 2005 contra sólo 67 ciudades en 1994 (Emelianoff, 1999), expresa implícitamente una demanda creciente de soluciones para pensar la ciudad del futuro. No cabe duda de que los geógrafos tienen competencias y saberes movilizables sobre esta problemática.

Con el objeto "ciudad", nos situamos de pleno en el campo de la geografía de las utopías. Desde tiempos muy remotos, lo urbano ha sido propicio a muchas visiones idealizadas y futuristas. Una de las referencias más conocidas es sin duda el libro de Thomas More, escrito en 1516, con un título sin ambigüedad, Utopía. Thomas More soñaba con un modelo de ciudad ideal, limitada a 6000 familias, liberada de sus impurezas, con un aire no corrompido, sin enfermedades y habitada por una sociedad perfecta regida por un sistema de vida comunitario. La ciudad-jardín de E. Howard, o la ciudad funcional de Le Corbusier, son otras expresiones de modelos urbanos utópicos. Así que contemplar la ciudad sustentable como una nueva utopía, no tiene nada sorprendente.

Cada nueva utopía, a lo largo de los tiempos, se fundó sobre el rechazo de un modelo existente (Holz, 2004, p. 111). En el caso de la ciudad sustentable, es el arquetipo de las grandes ciudades que está puesto en duda. La concentración de la población en las grandes urbes y la frenética extensión urbana del siglo XX levantan muchas críticas, entre las cuales están la consecutiva degradación de los espacios naturales periurbanos, la concentración de los contaminantes, la producción de grandes cantidades de desechos, el gasto de agua y de energía o el desarrollo sin fin de las redes de carreteras devoradoras de espacio (Mitchell, 
2004, p. 101-103).

¿Cuáles serían las características del contra-modelo de la ciudad sustentable? Sería una ciudad compacta, de dimensión mediana, con una débil huella ecológica, donde la naturaleza sería un elemento clave de la organización del espacio urbano, una ciudad también más justa, con menos fragmentación socio-territorial. Este nuevo proyecto parece ciertamente un Santo Grial difícilmente alcanzable. Pero sirve de punto de mira para orientar la acción sobre los territorios urbanos. Actuar sobre las dimensiones, las formas y la densidad de las ciudades, son sin duda aspectos que interesan directamente a la investigación geográfica (Wolff, 2004, p. 120-121).

La voluntad de reintroducir la naturaleza en la ciudad es quizás lo que más Ilama la atención en la utopía de la ciudad sustentable. Pues hasta hace poco, al menos en Europa, la naturaleza era percibida por la mayoría de los urbanistas como un anti-mundo, una negación de la ciudad, un territorio del vacío. Los vegetales en la ciudad, en sus diferentes formas (alineamiento de árboles, parques urbanos, etc.), lejos de ser reliquias de la naturaleza salvaje, estaban totalmente sometidos a lógicas urbanas y a menudo compuestos por especies alóctonas. De forma singular, a lo largo de los años 1990, la naturaleza se ha convertido en un elemento fundamental en todos los proyectos de recomposición de los espacios urbanos. Podemos ver ahí una rehabilitación del pensamiento higienista del siglo XIX, y más recientemente la influencia creciente de las teorías de la ecología urbana.

Los geógrafos pueden sin duda aportar sus competencias para enriquecer el debate sobre la ciudad sustentable, en particular sobre el juego complejo de las escalas territoriales implicadas. El acercamiento a esta problemática supone un cambio de escala a la hora de pensar la ciudad del futuro. En las grandes metrópolis, con límites cada vez más diluidos, es necesario plantear la cuestión de la sustentabilidad no solamente a la escala de los espacios propiamente urbanizados, sino a una escala regional y en la perspectiva más global del ordenamiento territorial (Mitchell, 2004, p. 106).

Pero esto no supone relegar en un segundo plano las escalas más locales (de la ciudad al espacio doméstico), sino más bien todo lo contrario. El ejemplo de la ciudad ecológica de Beddington, al sur de Londres, pone a la luz no solamente la factibilidad de tales realizaciones, sino también las perspectivas que representan estos proyectos para la investigación geográfica (Bovet et al., 2004). En el proyecto BedZED (Beddington Zero Energy Development), Ilevado acabo por el arquitecto Bill Dunster, con el apoyo de la ONG inglesa Bioregional, se abordaron cuestiones sumamente geográficas: la reconversión espacial de un antiguo basurero municipal, la utilización de materiales reciclables y ecológicos para el habitat, la definición de una zona de abastecimiento de 50 $\mathrm{km}$. alrededor de la ciudad para limitar los efectos negativos del transporte, la creación de una ciudad densa, el desarrollo de energías renovables, y todo eso con un cuidado espacial para la dimensión paisajística (edificios cubiertos de madera y con tejados vegetalizados). Esta claro que los geógrafos están todavía poco movilizados en torno a tales proyectos, y eso a pesar de la perspectiva estimulante de un diálogo renovado entre la geografía urbana, la geografía ambientalista y la geografía del ordenamiento territorial que podría aportar la investigación sobre la ciudad sustentable.

\section{La utopía del bosque sustentable}

También el tema de los bosques en los países del Sur ilustra claramente el carácter crucial del debate sobre el desarrollo sustentable, con una dimensión territorial que interesa directamente a la geografía. Aquí, dos modelos territoriales se enfrentan: el modelo de la partición y el modelo del multifuncionalismo. El modelo de la partición se funda sobre una concepción idealizada de la naturaleza (Robinson, 2002, p. 46; Arnould y Clément, 2004, p. 164). En resumen, consiste en separar por una parte los bosques "nativos", convertidos en santuarios (parques naturales), y 
definir el concepto de bosques naturales o nativos (Maser, 2000, p. 282-283). Si con ello se designa a los bosques libres de cualquier forma de humanización, sólo podría aplicarse a reductos forestales muy limitados del planeta. Si se trata de los bosques poco transformados por el hombre: ¿cuál sería el umbral de tolerancia de la presencia humana en tales bosques y qué provenir sustentable se les ofrece a los pueblos indígenas que habitan los bosques santuarios?

La puesta en marcha del modelo de la partición y sus consecuencias para los pueblos del bosque son bastante preocupantes. Los parques naturales dedicados a la conservación radical excluyen de hecho a las poblaciones instaladas en ellos, a veces desde tiempos remotos, ya que se les prohíbe cualquier forma de explotación o de recolección de los recursos forestales. Así, en África del Sur, los pueblos que viven en el reciente parque natural de Santa Lucía son considerados como ilegales y progresivamente desplazados (Guyot, 2003). En Bolivia, en el parque de Amboró que cuenta con 500.000 hectáreas de bosques tropicales, la política de conservación integral llevada a cabo ha marginalizado y empobrecido un poco más a los 40.000 indígenas que lo habitaban. Tales situaciones no se ven únicamente en los bosques tropicales. En Chile, los bosques templados de araucarias han sido incluidos en los parques naturales de Nahuelbuta y de Conguillío. No se trata de poner en duda el gran valor ecológico de estos bosques reliquias. Pero hay que preguntarse sobre el futuro de las comunidades indígenas pehuenche, cuyo nombre significa en mapundungun "la gente de los bosques de araucarias", puesto que ni siquiera pueden recolectar los piñones de araucarias, frutos tan importantes en su cultura alimenticia y en su modo de vida (Clément, 2005, p. 130-131). Por lo general, casi ninguna, ya que estas comunidades están consideradas como agente de degradación del entorno natural, y que no tienen la formación necesaria para participar en este turismo elitista. Cuando las comunidades pretenden desarrollar sus propias iniciativas de ecoturismo, como los mapuche en Chile, se enfrentan a numerosos obstáculos. Uno de ellos es el reconocimiento de sus proyectos como auténticamente ecoturísticos. En la guía de turismo chilena Turistel, el centro de ecoturismo Puaucho, llevado por las comunidades mapuche de la Laguna Budi, está presentado como un sitio de turismo «étnico», lo que claramente no tiene el mismo significado (Volle, 2005, p. 98).

En la utopía del desarrollo sustentable, éticamente no podemos dejar al borde del camino a una parte de la humanidad. La preservación del patrimonio biocultural de los pueblos indígenas no es menos legítima que la conservación de la biodiversidad. Son dos cuestiones intimamente asociadas, dos vertientes de una misma problemática (Rozzi et al., 2003, p. 53). Es indispensable tomar en cuenta las aspiraciones de las poblaciones locales. Para ello, se hace necesario inventar un modelo menos exclusivo, que tome en cuenta el deseo de un mejor vivir de las comunidades indígenas.

El modelo del multifuncionalismo, utopía que pretende conciliar las funciones ecológicas, económicas y sociales del bosque, abre perspectivas moralmente más justas, sin renunciar a una gestión equilibrada del patrimonio forestal. La reciente puesta en práctica del modelo multifuncional en Chile demuestra su aplicabilidad en los países del Sur (Barrué-Pastor, 2004, p. 44-46). Por ejemplo, la CODEFF (Comité Nacional Pro Defensa de la Fauna y de la Flora), ONG ambientalista chilena que cuenta con un departamento destinado a los bosques coordinado por Hernán Verscheure, desarrolla programas en este sentido (Wilcox, 1996, p. 100). Propone una orientación hacia un manejo sustentable de los bosques nativos, defendiendo una silvicultura suave, cumpliendo así con el doble objetivo de la preservación del bosque y de una mejoría de la situación de las comunidades indígenas (Otero, 1999).

La red internacional de Bosques Modelos, iniciada en 1992, y cuya sede se sitúa en Ottawa, persigue el mismo objetivo del multifuncionlismo. En 1998, se creó en Chile el Bosque Modelo de Chiloé, el primero de América del Sur (Hoffmann, 
2001, p. 289-290). Abarca unas 173.000 hectáreas de bosque siempreverde en el corazón de la isla. Llevar una gestión forestal participativa, incluyendo a las comunidades indígenas implicadas, desarrollar prácticas sustentables de explotación del bosque nativo, valorar el patrimonio cultural de las comunidades, mejorar sus condiciones de vida y preservar la biodiversidad, tales son las principales metas fijadas. Para ello, se ha fomentado una mejor valoración de los productos madereros, transformándolos localmente para romper con la exportación de materia primaria poco rentable. Se ha desarrollado la recolección y comercialización de subproductos forestales (plantas medicinales, avellanas silvestres, leña etc.). Por fin, se ha buscado diversificar las fuentes de ingresos con actividades artesanales (cestería, artesanía de madera, apicultura, etc.). A nuestro entender, este tipo de iniciativa cumple con los objetivos éticos, económicos y medioambientales contenidos en el concepto de desarrollo sustentable, lo que no es el caso del modelo de la partición. Aquí también, la geografía de las utopías tiene un espacio importante para desarrollar sus investigaciones $y$ formular nuevos planteamientos.

\section{CONCLUSIONES}

El análisis llevado a cabo del desarrollo sustentable nos permite concluir que este concepto constituye una ruptura, puesto que es portador de una crítica del modelo de desarrollo actual, responsable de mantener una situación de crisis económica casi permanente, de crear profundas desigualdades sociales y de ser destructor para el medio ambiente (Jollivet, 2001, p. 9).

Como hemos visto, lejos de ser un concepto nuevo, se inscribe en una filiación intelectual remota, cuyos orígenes se sitúan en la legislación medieval europea sobre los bosques. Los pensadores del siglo XIX aportaron una construcción teórica de aproximación a este concepto, que continuaría en los años 1970, en particular con las reflexiones de los economistas. Sin embargo, a pesar de ser una idea antigua, el desarrollo sustentable sí es revelador de los graves interrogantes que sacuden el mundo contemporáneo. Frente a las amenazas existentes sobre el medio ambiente, es necesario inventar nuevas relaciones, más equilibradas entre el hombre y su entorno. También la proyección hacia las generaciones futuras expresa de manera indirecta las inquietudes del presente, revelando implícitamente un rechazo de la cultura dominante de lo instantáneo. La sociedad contemporánea, hundida en la brecha de la actualidad y de la inmediatez de la información, tiene dificultad para articular la herencia del pasado con una visión clara de su porvenir.

El concepto de desarrollo sustentable Ilena en parte este vacío, puesto que conlleva un mensaje positivo, lo que explica en gran parte su éxito. De cara a la globalización y a sus peligros (ecológicos, sociales y culturales), el desarrollo sustentable ofrece una alternativa, la promesa de un futuro mejor, más ético y más respetuoso del medio ambiente. En cierta medida, esta utopía consagra el retorno de lo político y de lo social, frente al poder económico. Por lo contrario, la debilidad de este concepto reside en su carácter demasiado maleable y consensual. Por esta razón, es propicio a interpretaciones muy variadas que oscilan entre dos extremos, por un lado el de una lectura radicalmente conservacionista del desarrollo sustentable, y por otro el de una concepción ultraliberal en la cual, por ejemplo, el derecho a contaminar puede convertirse en una mercancía (Mitchell, 2004, p. 106-107).

Por ello, la movilización de la comunidad científica sobre la problemática del desarrollo sustentable representa tanto una necesidad como un desafío. Una necesidad, ya que para poder aportar soluciones a los grandes problemas contemporáneos, se precisa un acercamiento matizado y fundado científicamente. Un desafío, en el sentido de que el desarrollo sustentable nos conduce a pensar de manera innovadora, a inventar un nuevo pacto social entre el hombre y el medio ambiente, y a elaborar las bases de un renacimiento humanista para el siglo XXI. 
No cabe duda de que la geografía, en colaboración con otras disciplinas, pueda aportar una significativa contribución a esta problemática, ya que tiene competencias y saberes reconocidos sobre las cuestiones medioambientales. Pero no se trata de reciclar nuestra herencia disciplinaria sobre este tema. No sería una respuesta a la altura del desafío planteando. El desarrollo sustentable es más: nos abre un nuevo horizonte, un nuevo frente para la investigación, el de una geografía de las utopías. El nomadismo del concepto no puede ser un argumento válido para rechazarlo. ¿Hoy, quién pondría en duda el interés de otro concepto nómada, el de paisaje, cuya polisemia se ha revelado tan enriquecedora para la geografía? Sin embargo, para hacer del desarrollo sustentable un verdadero objeto de ciencia, es necesario precisar sus dimensiones espaciales y temporales, lo que constituye sin duda el talón de Aquiles de este concepto para la investigación geográfica.

\section{BIBLIOGRAFÍA}

ARNOULD P., CLÉMENT V. (2004). "Forêts et développement durable. De l'impératif de la durabilité à la question controversée des indicateurs", Historiens et Géographes, n³87, p.163-174.

BARRUÉ-PASTOR M. (EDIT., 2004). Forêts et développement durable au Chili. Indianité mapuche et mondialisation, Toulouse, Presses Universitaires du Mirail, 287 p.

BERTRAND G., BERTRAND C. (2002). Une géographie traversière. L'environnement à travers les territoires et les temporalités, Paris, Arguments, 311 p.

BOVET PH., JEANVOINE N. (2004), «Ecologiques de $A$ à Zed: une cité qui préfigure les villes écologiques de demain», La Maison Écologique, n²1, p. 28-31. BRUNDTLAND REPORT (1987), Our Comon Future, ONU, Comisión para el Medio Ambiente y el Desarrollo, Oxford, Oxford University Press, 400 p.
CLÉMENT V. (2002). De la marche-frontière au pays-des-bois. Forêts, sociétés paysannes et territoires en Vieille Castille (XI ${ }^{e}-X X^{e}$ siècle), Madrid, Casa de Velázquez, 374 p.

CLÉMENT V. (2005). "Peuple de la forêt, peuple de la frontière. Identité et territoire des indiens pehuenches dans l'ancienne marche araucane (XVIXXle siècle)", Mélanges de la Casa de Velázquez, $n^{\circ} 35(2)$, p. 125-145.

COURNOT A.A. (1861). "Traité de l'enchaînement des idées fondamentales dans les sciences et dans I'histoire", en Oeuvres complètes, tomo 3, Paris, CNRS, Librairie Vrin.

DUCROUX A.M. (EDIT., 2002). Les nouveaux utopistes du développement durable, Paris, Autrement, 342p.

EMELIANOFF C. (1999). La ville durable, un modéle émergent. Géoscopie du réseau européen des villes durables, Universidad de Orléans, tesis doctoral inédita, $746 \mathrm{p}$.

GUYOT S. (2003). "Les conflits environnementaux à Santa Lucia, entre protection et exclusion (Afrique du Sud)", Annales de Géographie, n634, p. 608627.

HOFFMANN A. (2001), Enciclopedia de los bosques chilenos. Conservación, biodiversidad, sustentabilidad, Santiago de Chile, Defensores del Bosque chileno, Colección Voces del Bosque, 351p.

HOLZ J.M. (2004). "La ville durable: une nouvelle utopie ?", Historiens et Géographes, n³87, p. 109113.

JOLLIVET M. (EDIT., 2001). Le développement durable, de l'utopie au concept. De nouveaux chantiers pour la recherche, Paris, Elsevier, Natures, Sciences, Sociétés, 288 p.

KALAORA B. (2004). "Du développement au développement durable. Un défi pour les sciences sociales", Historiens et Géographes, $n^{\circ} 387$, p. 157-162. 


\section{Revista CUHSO volumen $10 n^{\circ} 2$}

MARSH G.P. (1864). Man and Nature: or Physical Geography as Modified by Human Action, Cambridge, Harvard University Press.

MARTIN J.Y. (EDIT., 2002). Développement durable? Doctrine, pratiques, évaluations, Paris, IRD, 344 p.

MASER C. (2000). Sustainable Forestry. Philosophy, Science and Economics, Londres, Santa Lucie Press, 373 p.

MEADOWS D.H. (1972). The Limits to Growth: A Report of the Club of Rome's Projet on the Predicament of Mankind, New York, Universe Books.

MILL J.S. (1848). Principes d'économie politique, Paris, Dalloz.

MIOSSEC A., ARNOULD P., VEYRET Y. (EDIT., 2004). "Vers une géographie du développement durable", UGI-CNFG, edición especial de la revista Historiens et Géographes, n³87, 260 p.

MITCHELL G. (2004). "Forecasting Urban Future : A System Analytical Prespective on the Development of Sustainable Urban Regions", en Purvis M., Grainger Alan (edit.), Exploring Sustainable Development. Geographical Perspectives, Londres, Earthscan, capítulo 5, p. 99-127.

O'CONNOR MARTIN (1995). “La reciprocité introuvable. L'utilitarisme de John Stuart Mill et la recherche d'une éthique de la durabilité", Economie appliquée, n48-2, p. 271-304.

OTERO L. (1999). Manual de manejo sustentable de bosques nativos para pequeños propietarios, Santiago de Chile, CODEFF-Amigos de la Tierra, $83 p$.

PISANI J.A. (2005). "Sustainable Development. Historical Roots and Evolution of the Concept", en Agnoletti M. (edit.), History and Sustainability, Actas de la II $^{\text {a }}$ Conferencia Internacional de la Sociedad Europea de Historia Medioambiental (Florencia, 16-19 febrero 2005), p. 28-37.
REAL ACADEMIA DE LA HISTORIA (1863). Cortes de los Antiguos Reinos de León y de Castilla, Madrid, $556 \mathrm{p}$.

ROBIC M.C., MATHIEU N. (2001). "Géographie et durabilité: redéployer une expérience et mobiliser de nouveaux savoir-faire", en Jollivet (edit.), Le développement durable, de l'utopie au concept. De nouveaux chantiers pour la recherche, Paris, Elsevier, Natures, Sciences, Sociétés, p. 167-190.

ROBINSON G.M. (2002). "Nature, Society and Sustainability", en Bowler I.R, Bryant C.R., Cocklin C. (edit.), The Sustainability of Rural Systems. Geographical Interpretations, Dordrecht, Kluwer Academic Publishers, capítulo 3, p. 35-57.

ROZZI R. ET AL. (2003), «Conservación biocultural y ética ambiental en el extremo austral de América: oportunidades y dificultades para el bienestar ecosocial», en Figueroa E., Simonetti J., Globalización y biodiversidad: oportunidades y desafíos para la sociedad chilena, Santiago de Chile, Editorial Universitaria, p. 51-85.

SACHS I. (1977). Pour une économie politique du développement, Paris, Flammarion.

STENGERS I. (EDIT., 1987). D'une science à l'autre. Des concepts nomades, Paris, Seuil.

THOMPSON I.H. (2000). "The ethics of sustainability", en Benson J.F., Roe M.H. (edit.), Landscape and Sustainability, Londres, Spon Press, capítulo 2, p. 12-32.

VIVIEN F.D. (2001). "Histoire d'un mot, histoire d'une idée: le développement durable à l'épreuve du temps", en Jollivet (edit.), Le développement durable, de l'utopie au concept. De nouveaux chantiers pour la recherche, Paris, Elsevier, Natures, Sciences, Sociétés, p. 19-60.

VOLLE A. (2005). Quand les Mapuches optent pour le tourisme. Regards croisés sur le développement au Chili, Paris, L'Harmattan, colección Tourisme et Société, 227 p. 


\section{UNIVERSIDAD CATÓLICA DE TEMUCO}

WILCOX K. (1996), Chile's Native Forests. A Conservation Legacy, Redway, Ancient Forest International, NW Wild Books, 148 p.

WOLFF J.P. (2004). "Morphologie urbaine et développement urbain durable", Historiens et Géographes, n³87, p. 115-122. 